\title{
Soziale Marktwirtschaft 2.0 - eine Replik
}

\author{
SEBASTIAN THIEME
}

Der Zustandsbeschreibung des deutschen Sozialstaats, die Florian Josef Hoffmann kürzlich in den WSI-Mitteilungen lieferte, lässt sich durchaus zustimmen: Der Ordoliberalismus - verstanden im Sinne der Garantie individueller Freiheit im Rahmen liberaler Rechtsstaatlichkeit gegen „zu viel staatliche Regulierung", also als Freiheit in Ordnung (im Sinne von Franz Böhm u. a.) - betreibe „in Deutschland noch heute einseitig [...] die Verdrängung der Solidarsysteme der Arbeiterschaft, der Gewerkschaften“` Seine Ausführungen zur Sozialen Marktwirtschaft sind aus ideengeschichtlicher Sicht allerdings zu ergänzen. Das gilt vor allem deshalb, weil Hoffmann den Bezug zu Alfred Müller-Armack sucht und dabei nicht nur einzelne Missverständnisse entstehen, sondern der Blick auf Müller-Armack eigentlich auch eine andere Sicht auf die Soziale Marktwirtschaft ermöglicht, als sie Hoffmann in seinem Beitrag bietet.

\section{Soziale Marktwirtschaft als Stilidee}

Zunächst ist darauf hinzuweisen, dass Alfred Müller-Armack nicht nur aus religionssoziologischer Sicht betrachtet werden sollte, sondern ebenfalls als geprägt durch die Idee der Wirtschaftsstile. Das gilt es vor allem im Blick zu behalten, wenn Hoffmann ganz richtig feststellt, dass die Historische Schule „Zwischen den Weltkriegen in der Bedeutungslosigkeit" versank: Trotzdem sich die Vertreter der Wirtschaftsstile vom alten Wirtschaftsstufen-Denken der Historischen Schule distanzierten, so stand das Denken in Wirtschaftsstilen dennoch unter der Prägung der Historischen Schule, was z. B. die ganzheitliche Betrachtung der gesellschaftlichen Lebensbereiche (Politik, Wirtschaft usw.) anbelangte. In dieser Tradition steht auch Müller-Armack. Die mittlerweile als Standardwerk zur Sozialen Marktwirtschaft bekannte Schrift von Friedrun Quaas bestimmt Soziale Marktwirtschaft folglich auch als offene Stilidee. ${ }^{2}$ Doch was bedeutet das konkret?

Mit Bezug auf Müller-Armack heißt das vor allem, die Soziale Marktwirtschaft in „Gesamtordnungen zu denken". ${ }^{3}$ Die Bereiche Wirtschaft, Politik, Soziales usw. sollten in ihrer Gesamtheit beachtet werden; der Mensch sei „im Doppelaspekt seiner ökonomischen wie geistigen Lebensform “( ${ }^{\text {“( }}$ anzusehen. Ferner müsse der Mensch mit seinem Handeln als geschichtlich begriffen werden. Zwar wird der Mensch in eine gegebene Situation hineingeboren, doch vermag er sie zu verändern. Aus der Geschichtlichkeit des Wirtschaftsstils heraus lassen sich deshalb keine immergültigen Handlungsrezepte vorgeben: Jede Situation ist einmalig! Darauf basiert die Offenheit der Stilidee der Sozialen Marktwirtschaft. Sie wäre jedoch unzureichend beschrieben, wenn nicht noch die Idee der Sozialen Irenik Erwähnung fände.

\section{Soziale Irenik}

Dabei handelt es sich um ein Charakteristikum, das in den Debatten und in der Literatur zur Sozialen Marktwirtschaft leider viel zu kurz kommt und aus dessen Ausblendung häufig ein recht zentrales Missverständnis resultiert. Fälschlich werden oft die obersten sittlichen Ziele der Sozialen Marktwirtschaft - Freiheit und soziale Gerechtigkeit $^{\bullet}$ - gegeneinander aufgewogen. Statt eines solchen Aufrechnens steht die Soziale Irenik, die Müller-Armack in einem Aufsatz von 1950 beschrieb, für eine Versöhnung der unterschiedlichen gesellschaftlichen Standpunkte: Die jeweils anderen Sichtweisen sind anzuerkennen und Kompromisse zu schließen, ohne aber den charakteristischen Kern des eigenen Andersseins aufzugeben. ${ }^{\bullet}$ Es geht also um ein Spannungsverhältnis, in dem sich die Menschen in einer Sozialen Marktwirtschaft vor dem Hintergrund ihrer geschichtlich einmaligen Situation immer wieder aufs Neue zu positionieren haben. Was Freiheit und soziale Gerechtigkeit bedeuten, das ist demnach ständig neu zu definieren.

Insofern geht es etwas fehl, wenn Hoffmann die obersten Ziele der Sozialen Marktwirtschaft auf die Begriffe solidarisches Wirtschaften und Ordoliberalismus überträgt, beides in jeweils das Prinzip der Solidarität und das Prinzip der Subsidiarität transformiert und fordert, es müsse ein „Gleichgewicht [sic!] von solidarischer Wirtschaft und Ordoliberalismus, von Solidarität und Subsidiarität“ herbeigeführt werden. Denn das Konzept der Sozialen Marktwirtschaft zielt nun gerade nicht auf eine „eindeutige und kollisionsfreie Kombination der beiden Ansätze" ab, sondern - im Gegenteil auf einen Gesellschaftsentwurf, der Kollisionen und Meinungsverschiedenheiten, kurz: ein gesellschaftliches Spannungsverhältnis, ausdrücklich zulässt. Hinsichtlich der gesellschaftlichen Ent-
Dieses und alle anderen Zitate von Hoffmann stammen aus Hoffmann, F. J. (2011): Soziale Marktwirtschaft - neu definiert, in: WSI-Mitteilungen 64 (10), S. 556-558, http://www.boeckler. de/wsimit_2011_10_hoffmann.pdf.

2 Vgl. Quaas, F. (2000): Soziale Marktwirtschaft: Wirklichkeit und Verfremdung eines Konzepts, Bern/Stuttgart/Wien.
3 Müller-Armack, A. (1981[1973]): Die wissenschaftlichen Ursprünge der Sozialen Marktwirtschaft, in: Dürr, E. (Hrsg.): Genealogie der Sozialen Marktwirtschaft, Ausgewählte Werke von AIfred Müller-Armack, Bern, S. 183.

4 Müller-Armack (1981[1973]): Ursprünge, a. a. O., S. 183.

(5 Vgl. Müller-Armack, A. (1981[1948]): Vorschläge zur Verwirklichung der Sozialen Marktwirtschaft, in: Dürr, E. (Hrsg.): Genealogie der Sozialen Marktwirtschaft, Ausgewählte Werke von Alfred Müller-Armack, Bern, S. 90-91.

6 Vgl. Müller-Armack, A. (1981[1950]): Soziale Irenik, in: Dürr, E. (Hrsg.): Religion und Wirtschaft: Geistesgeschichtliche Hintergründe unserer europäischen Lebensform, Bern/Stuttgart, S. 559-578; Thieme, S. (2011): Betreff Rückschau: Die Evolutionäre Ethik von Hans Mohr, in: Erwägen Wissen Ethik 21 (4), S. 586-587. 
scheidungsprozesse lässt sich das als Forderung nach einem offenen Diskursklima interpretieren, das geradewegs in die Integrative Wirtschaftsethik eines Peter Ulrich führt. ${ }^{\boldsymbol{0}}$ In diesem Sinne kommt der Bestrebung nach Mitbestimmung und Demokratisierung innerhalb der Sozialen Marktwirtschaft eine wichtige Funktion zu.

\section{Zweidimensionale Subsidiarität}

Ferner ist anzumerken, dass Hoffmann dem Prinzip der Subsidiarität zwar ganz klar eine soziale Komponente zuspricht, dann jedoch das individuelle eigenverantwortliche „Handeln vor das Handeln des Staates" stellt. Dadurch wird jedoch dem weit verbreiteten Zerrbild Vorschub geleistet, das den Fokus recht einseitig auf das Interventionsverbot des Staates legt. Deshalb sei ausdrücklich auf den zweidimensionalen Charakter der Subsidiarität hingewiesen: Denn gleichzeitig zum Interventionsverbot ist Subsidiarität als Gebot zur Hilfe zu verstehen. ${ }^{8}$ Anders formuliert: „Subsidiarität greift ergänzend als Hilfe zur Selbsthilfe dann ein, wenn die Handlungskapazitäten geringerer Stufe erschöpft sind oder in absehbarer Weise nicht ausreichen “ $\odot$ Subsidiarität steht also für einen Anspruch (!) auf Unterstützung, was in der - von Hoffmann zu Recht kritisierten - ordoliberalen Einseitigkeit meist völlig unter den Tisch fällt.

\section{Ordnungsrahmen, Akzeptanz und Neoklassik}

Unbedingt ist Hoffmann zuzustimmen, dass eine Soziale Marktwirtschaft ohne einen rechtlichen Ordnungsrahmen nicht denkbar wäre.
Die Fixierung auf diesen Ordnungsrahmen kann allerdings dazu führen, gegenüber jenen Prozessen blind zu sein, die die Soziale Marktwirtschaft als Stilidee offen halten sollen. Das Ergebnis ist dann ein Ordnungsrahmen, aus dem wirtschaftspolitische Maßnahmen hervorgehen, denen es an Akzeptanz und ethischer Legitimation fehlt, wofür die Hartz-Reformen, die im Wesentlichen unter Ausschluss der Expertise der Sozialverbände entworfen wurden, ein gutes Beispiel abgeben. ${ }^{(1)}$

Wenn es um die Umsetzung der Sozialen Marktwirtschaft geht, tritt häufig noch ein weiteres Missverständnis auf, das einer Klärung bedarf. So schreibt Hoffmann von einem „überwältigenden Erfolg der Sozialen Marktwirtschaft" und von einem „bisher unbekannten, ungeahnten wirtschaftlichen Wohlstand”. Kritische Leser mögen hier geneigt sein zu fragen, worauf sich das bezieht? Schließlich ist mit Egon Tuchtfeld der Stilverfall der Sozialen Marktwirtschaft bereits in den 1960er Jahren zu beobachten. ${ }^{(1)}$ Os wald von Nell-Breuning sah den Stilverfall sogar schon in der Implementierungsphase aufkommen, also zwischen 1948 und 1950. ${ }^{12}$

Einer weiteren Ergänzung bedarf die Behauptung, „[d]ie ordoliberalen Gedanken“ wären „in der Wissenschaft von den Neoklassikern verdrängt" worden. Denn Ordoliberalismus und ökonomische Neoklassik sind keine Gegensätze: Wirtschaftspolitische Empfehlungen laufen in aller Regel auf einen deutlich neoklassisch ge prägten Ordoliberalismus hinaus, was sich z. B. an den Vorschlägen der fünf „Wirtschaftsweisen zum Arbeitsmarkt ablesen lässt. ${ }^{\circledR}$ Die Ordnungsethik von Karl Homann liefert ein weiteres Beispiel dafür. ${ }^{(*)}$

\section{Fazit}

Abschließend bleibt festzuhalten, dass die „Auflösbarkeit” bzw. das „Gleichgewicht” der von Hoffmann als gegensätzlich beschriebenen Ideen (Ordoliberalismus und soziales Wirtschaften) nicht wirklich Ziel der Sozialen Marktwirtschaft war. Es ging um Kompromisse, das sprichwörtliche Ziehen am gleichen Strang, aber gleichzeitig auch um das Aufrechterhalten gesellschaftlicher Spannungsverhältnisse. Müller-Armack sprach diesbezüglich auch von einem „dialektischen Verhältnis“. ${ }^{\circledR}$ Das eine solche offene Konzeption unscharf ist, sollte klar sein. Aber diese Offenheit lässt sich nicht nur als Mangel, sondern - im Gegenteil - als Stärke der Stilidee begreifen. Es ist eben kein konkretes $\mathrm{Pa}$ tentrezept, keine statische Allround-Lösung. Mit der Sozialen Irenik verfügt die Soziale Marktwirtschaft aber über ein Element, mit dem sie aus sich selbst heraus Neues schafft. Sie trägt den Keim der Erneuerung praktisch in sich. Das macht sie zu einem Konzept, das sogar den modernen evolutionsökonomischen Ansätzen gerecht wird und sich gleichzeitig an die nicht weniger evolutorische wirtschaftsethische Konzeption der Integrativen Wirtschaftsethik anschlussfähig hält. ${ }^{\left({ }^{\circ}\right.}$ Diese im Kern evolutorische Perspektive stellt die Notwendigkeit eines echten Diskursklimas, der Partizipation und der Demokratisierung unserer Lebensbereiche in den Vordergrund. Einzelne dieser Elemente werden gerade wieder neu erprobt und eingeklagt (Stuttgart 21, Volksentscheide/ Forderung nach mehr politischer Mitbestimmung usw.) Gewerkschaften wie Parteien können diese Entwicklungen beflügeln und auf diese Weise die Implementierung einer Sozialen Marktwirtschaft 2.0 in greifbare Nähe rücken lassen.
$(7$ Vgl. Ulrich, P. (2008): Integrative Wirtschaftsethik: Grundlagen einer lebensdienlichen Ökonomie, Bern et al.

8 Vgl. Quaas, F. (2000): Wirklichkeit und Verfremdung, a. a. O, S. 338-339.

(9 Waschkuhn, A. (1995): Was ist Subsidiarität?, Opladen, S. 170.

(10) Vgl. Thieme, S. (2011): Sozialstaat und ethische Legitimität, MPRA Paper (30324), München.

(1) Vgl. Quaas, F. (2000): Wirklichkeit und Verfremdung, a. a. O, S. 117.

(12) Vgl. ebd., S. 346 .

(13 Vgl. Sachverständigenrat zur Begutachtung der gesamtwirtschaftlichen Entwicklung (2010):
Chancen für einen stabilen Aufschwung: Jahresgutachten 2010/11, Wiesbaden.

(4) Homann, K. (2008): Was bringt die Wirtschaftsethik für die Ethik?, Wittenberg-Zentrum für Globale Ethik, Diskussionspapier 2008-04, Wittenberg.

(15 Vgl. Quaas, F. (2005): Soziale Marktwirtschaft: Einführung, in: Hasse, R. H./Schneider, H./Weigelt, K. (Hrsg.): Lexikon Soziale Marktwirtschaft, Paderborn et al., S. 360-363.

(16) Vgl. Quaas, F. (2009): Der Wirtschaftsstil der Sozialen Marktwirtschaft aus evolutorischer Perspektive, in: Aßländer, M./Ulrich, P. (Hrsg.): 60 Jahre Soziale Marktwirtschaft. Illusionen und Reinterpretationen einer ordnungspolitischen Integrationsformel, St. Galler Beiträge zur Wirtschaftsethik, Bd. 44, Bern/Stuttgart/Wien, S. 119-145.

\section{AUTOR}

SEBASTIAN THIEME, Dr., promovierte an der Wirtschaftswissenschaftlichen Fakultät der Universität Leipzig über das Subsistenzrecht als Moralprinzip und ist Fellow bei der MeM - Denkfabrik für Wirtschaftsethik. Arbeitsschwerpunkte: Wirtschaftsethik, ökonomische Ideengeschichte und Menschenfeindlichkeit in der Ökonomik.

sebastian.thieme@uni-leipzig.de 